Artículo original

\title{
DESARROLLO DE UN SISTEMA INTEGRAL DE GESTIÓN (SIG) PARA MEJORAR LA GESTIÓN DE PROYECTOS EN OBRAS DE SANEAMIENTO EN GOBIERNOS LOCALES.
}

Development of an integrated management system (gis) to improve the management of projects in sanitation works in local governments.

\section{RESUMEN}

El objetivo es desarrollar un sistema integral de Gestión de los procesos para el cliente (entidad pública), todo ello desde la compatibilización del expediente técnico terminado y aprobado para luego dar pase a la elaboración de los TDRs de supervisión y construcción desde donde se colocan los requisitos necesarios que requiere el cliente para poder gerenciar el proyecto planificando y controlando las acciones necesarias para llegar a tener un buen control del proyecto. Se usó un conjunto de formatos estructurados para obtener información relevante de un proyecto el cual después pueda ser manejado de manera versátil en la simulación del sistema integral de gestión. La relación entre la formatología con información estructurada que ayuda a asegurar la calidad de los procesos y procedimientos de gestión reduce de manera significativa los tiempos de planificación y control de procesos y procedimientos. Mala ejecución de obra en tiempo, costo y especificaciones está ligado básicamente a la inadecuada supervisión y ejecución de obra en los procesos constructivos por parte de las empresas. No cuentan un método o sistema para llevar adecuadamente la obra Los en ese sentido que el conjunto de formatos estructurados que conforman el sistema integral de gestión reduce de forma significativa los procesos, procedimientos, antes descritos.

Palabras clave: Supervisor de obras, contratista, Gestión, PMBOK, Aseguramiento de calidad,

Procesos, Procedimientos, Alcance, Tiempo, costos, Entidad pública.

\section{ABSTRACT}

The document that is presented addresses the problem of frequent deviations that occur in the management of projects, poor execution of work in time. Cost and specifications, which finally marks the planned utilities for the projects, as well as the consequent dissatisfaction of the clients that as a client usually does not perform a control task but rather leaves all the activities to the supervisor and this entails no Take action before the events occur. All this is basically linked to the inadequate supervision and execution of work in the construction processes by the companies, that is, do not count or apply a method, system to carry the work properly.

The main objective of this work is to develop an integrated system of Process Management for the client (public entity), all from the compatibility of the technical file completed and approved to then move on to the elaboration of the TDRs supervision and Construction from where the necessary requirements are placed that the client requires to be able to manage the project planning and controlling the necessary actions to get a good control of the project requesting to the supervisor and contractor basic information that allow to take preventive and corrective actions in such a way that Can have a positive impact in the course of the Project.

Keywords: Project Supervisor, Contractor, Management, PMBOK, Quality Assurance, Processes, Procedures, Scope, Time, Costs, Public Entity.

\section{INTRODUCCIÓN}

El agua y el saneamiento tienen una importancia trascendental para la vida y salud de todas las personas. La ONU fijó entre los objetivos del Milenio que para el 2015 se debía reducir a la mitad el número de personas que carecen de acceso al agua potable y del servicio básico de saneamiento. EI INEI precisa que más de 4 millones de peruanos carecen de agua potable y más de 9 millones no tienen servicio de desagüe conectado a la red pública. Existe un gran número de obras paralizadas e inconclusas a nivel nacional, así como de otras culminadas pero que presentan graves deficiencias para su funcionamiento, mermas en su calidad, incompatibilidad de planos, con mayor presupuesto de lo planificado, no se consigue el alcance esperado; lo cual es motivo de investigación. Actualmente no se cuentan con sistemas de gestión de calidad eficientes que minimicen riesgos, se cumplan con los plazos a través de un control efectivo sin mermas de calidad del producto. La gestión de las obras es otro factor importante, tanto para el éxito de la misma(Alcina, Jorge, 2006). El desarrollo de un sistema de gestión de calidad para proyectos de saneamiento mejora la revisión de los expedientes técnicos antes del

\footnotetext{
1 Magíster en Ingeniería Civil con mención en Gerencia de la Contrucción
} 
proceso de licitación para posteriormente cerrar adecuadamente el alcance, el cual minimiza los riesgos para una eficiente ejecución, todo ello basado en estándares reconocidos como buenas prácticas en la gestión de los proyectos. Ya Farfán en Guatemala, refería en su investigación que un modelo básico ofrece el marco legal necesario para la rectoría de la función de abastecer de agua potable, establece las responsabilidades y los responsables de las acciones a realizar. Todo ello con el fin de involucrar las políticas y estrategias del sector(Farfan Cruz, J, 2005). Hernández, afirmaba que la sostenibilidad económica depende de la aplicación de tarifas que les permita generar los recursos financieros para mantener funcionando los sistemas. Para ello debemos de saber que se tiene que contar con un adecuado Proyecto de Trabajo(Hernández Rodríguez, 2013). El Ciclo de un Proyecto contempla las Fases de Preinversión, Inversión y Postinversión.

El concepto de expediente técnico tiene distintos significados y contenidos como suele suceder en los conceptos profesionales. La ingeniería tiene al expediente técnico como un documento vinculado a la ejecución de obra más o menos detallado dependiendo de lo que se construya, tanto es así que puede tener distintas aproximaciones con contenidos disimiles pero que van colaborando hacia la consecución de un objetivo final: la obra. Es el conjunto de documentos que incluye: memoria descriptiva, especificaciones técnicas, planos de ejecución de obra, metrados, valor referencial (presupuesto de obra), análisis de precios unitarios, calendario valorizado de avance de obras, fórmulas polinómicas. Adicionalmente, puede incluir si así se determina técnicamente: estudios de suelos, estudio geológico, estudio de impacto ambiental, estudio básico de ingeniería, u otros complementarios.

En la obra pública dos son los sistemas administrativos que regulan la ejecución de proyectos en el Estado, el Sistema Nacional de Inversión Pública y el de contrataciones del Estado(LEY No 30225 y D.S. N³50-2015-EF, s. f.). Cada Entidad Pública que conforma el Estado tiene a su cargo una serie de competencias, las cuales se encuentran dirigidas a la satisfacción de determinadas necesidades de la sociedad. En la actualidad las organizaciones se encuentran inmersas en entornos y mercados competitivos y globalizados, en los que toda organización que desee tener éxito tiene la necesidad de alcanzar buenos resultados empresariales; para esto, las organizaciones necesitan gestionar sus actividades y recursos con la finalidad de orientarlos hacia la consecución de los mismos, siendo necesario adoptar herramientas y metodologías que permitan a la organización configurar su Sistema de Gestión. Un Sistema de Gestión, ayuda a una organización a establecer las metodologías, las responsabilidades, los recursos, las actividades, etc., que le permitan una gestión orientada hacia la obtención de buenos resultados, o la obtención de los objetivos establecidos. La ingeniería del valor es un sistema en el que se analizan y aplican medidas correctivas a un bien o servicio en el cual se busca maximizar los recursos y disminuir los costos de producción. La Gestión de Proyectos Alineados al PMBOK del P.M.I , según el P.M.I y su estándar PMBOK(PMBOK del P.M.I, 2013), es la aplicación de conocimientos, habilidades, herramientas y técnicas a las actividades del proyecto para cumplir con los requisitos. Esto se logra con la aplicación adecuada de los procesos de dirección de proyectos, agrupados en los 5 grupos de procesos que son: Iniciación, Planificación, Ejecución, Seguimiento y Control, y Cierre.Todo gerente de proyecto debe velar porque en el ciclo de vida de su proyecto estén documentados esos aspectos(Cameron, K.S., and Whetten, D.A., 1983).

\section{OBJETIVOS}

a. Diseñar los procesos y procedimientos para la correcta revisión del expediente técnico de las obras de saneamientos por parte del gestor del proyecto.

b. Formular términos de referencia estándares para los procesos licitatorios para la ejecución y supervisión de las obras de saneamiento para una adecuada administración del contrato.

c. Desarrollar los procesos y procedimientos para la gestión de la planificación y control de los proyectos desde el enfoque del gestor del proyecto que contratará empresas para la construcción y supervisión de obras de saneamiento.

\section{METODOLOGÍA}

Tipo de investigación aplicada, no experimental. El ámbito de la investigación abarca a los gobiernos locales que hayan ejecutado proyectos de inversión pública afines con temas de agua y saneamiento entre los años 2013 al 2016: Municipalidad Provincial de Tacna, Municipalidad Provincial de Jorge Basadre, Municipalidad Provincial de Candarave y Municipalidad Provincial de Tarata. De estas municipalidades se entrevistaron a 30 profesionales involucrados en proyectos de saneamiento. Se utilizaron los formatos gestión para la revisión de expedientes, formatos de gestión para términos de referencia de contratación del ejecutor y supervisor y formatos para la gestión de proyectos desde el punto de vista de gerenciador público. 


\section{RESULTADOS}

\section{DIAGNÓSTICO SITUACIONAL}

Entrevista a los profesionales involucrados en proyectos de saneamiento de la región Tacna. El $100 \%$ de los profesionales encuestados son ingenieros. El $36.6 \%$ se encuentra en el rango maximo de 5 años de experiencia involucrados en

proyectos de saneamiento, y un $20 \%$ de profesionales con mas de los 15 años de experiencia profesional. El $\mathbf{7 6 . 7 \%}$ ha participado y/o elaborado bases, TDR, para obras de saneamiento. El $90 \%$ ha participado en la construcción de obras de saneamiento. El $76.67 \%$ ha participado en supervisión de obras. El $63.3 \%$ recibierón expedientes compatibilizados en un $75 \%$.

El $70 \%$ afirma que el mayor porblema se encuentra en la compatibilidad de los expedientes tecnicos. El $63.3 \%$ piensa que cuando se elaboran los TDRs se describe claramente el proposito que persigue la consultoria y el objetivo es claro. El $60 \%$ piensa que cuando se elaboran los TDRs no estan claramente definidas las actividades, tareas del consultor y no tiene en cuenta los entregables por metas (Grafico.1).

I $70 \%$ piensa que cuando se elaboran los TDRs no se definen con presición y tiempo los entregables que el consultor debera presentar a la entidad contratante. El 63.3\% afirma que cuando se elaboran estos no se indica claramente el plazo que tendra la entidad contratante para revisar aprobar o rechazar los informes o productos. El $80 \%$ afirma que cuando se elaboran los TDRs no se tiene en cuenta los entregables de gestión (Grafico.2)

El $90 \%$ afirma que cuando se elaboran los TDRs no se especifican las situaciones que pueden constituirse en conflictos de intereses. El 70\% afirma que cuando se elaboran los TDRs no se incluyen los parrafos adecuados para salvaguardar la informacion confidencial. El $53 \%$ afirma que cuando se elaboran los TDRs no se tiene mucho en cuenta el estudio de mercado que verifique la viabilidad de estudios (Grafico.3).

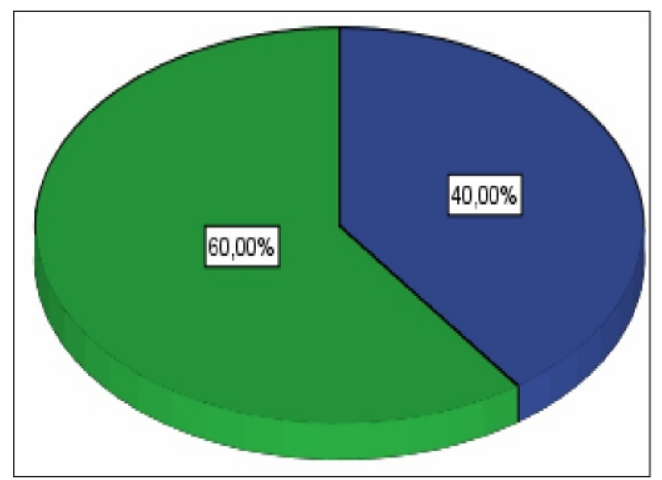

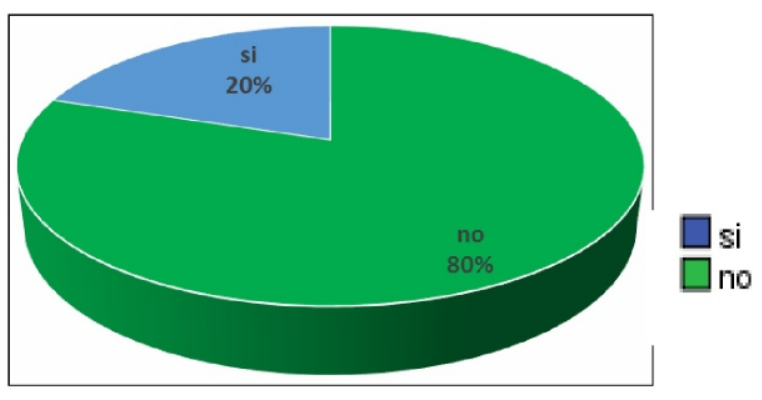

Grafico 2: Entregables de gestión

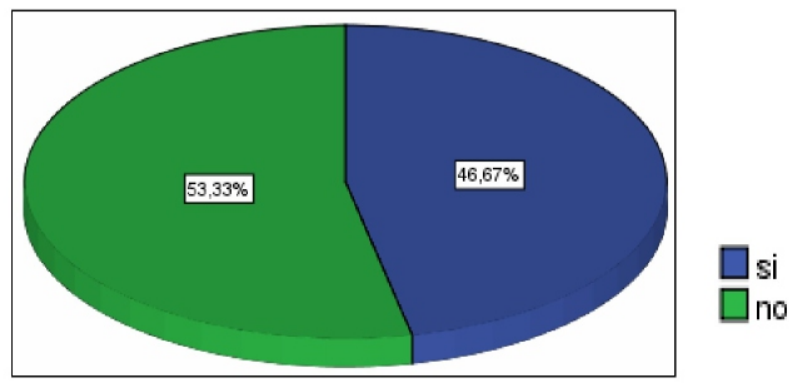

Grafico. 3: Estudios de mercado

El $80 \%$ afirma que los procedimientos tanto de revisión, compatibilización y/o aprobación del expediente técnico no son los más adecuados. El $73 \%$ afirma y cree que las entidades públicas no definen adecuadamente el alcance que deben realizar las consultoras de expedientes para el sector público. El $43 \%$ afirma que de los expedientes revisados y observados la cantidad de consultas se encontraba entre 0 y 30 , el $30 \%$ observó entre 30 y 70 consultas y observaciones. El $86.7 \%$ refiere que contando con un sistema de calidad alineada a un modelo de estándar internacional de gestión solucionaríamos varios problemas en la gestión de la formulación de TDRs. El $96.7 \%$ afirma que para gerenciar la ejecución de un proyecto de saneamiento desarrolla el Plan de Gestión del Proyecto, el $56.7 \%$ la Estructura Desglose del Trabajo - EDT, el 83.3\% desarrolla el Organigrama, el $76.7 \%$ la Matriz de Responsabilidades, el $53.3 \%$ la Matriz de Comunicaciones, el $66.7 \%$ el Plan de Gestión Documental, el $80 \%$ el Enunciado del Alcance, el $63.3 \%$ Aseguramiento de Calidad, el $73.3 \%$ el Registro de inspección de Seguridad, el $86.7 \%$ la Matriz de Riesgos, el $76.7 \%$ el Registro de Inspección de Medio Ambiente, el 83.3 el Registro de Calidad de los Materiales, el $70 \%$ el Registro de

Grafico. 1: Elaboración de los TDRs. 
Inspección de Procesos y el 80 \% informes de Desempeño del Proyecto.

Respecto a documentos a solicitar al contratista, el $90 \%$ afirma que para gerenciar la ejecución de un proyecto de saneamiento debería solicitar al contratista el Plan de la gestión integral, el $56.7 \%$ tableros de control de tiempo y costo, el $66.7 \%$ el Plan de respuesta a riesgos de Obra.
Respecto a qué documentos debería solicitar a la supervisión cuando desarrolla la gerencia de proyecto de saneamiento, el $86.7 \%$ afirma que para gerenciar la ejecución de un proyecto de saneamiento debería solicitar a supervisión Planes de Aseguramiento de Calidad de la Obra, el $93.3 \%$ Registros de Inspección de Procesos, el $56.7 \%$ el listado de solicitudes de cambios y el $63.3 \%$ las lecciones aprendidas.

\section{PROPUESTA DE UN SISTEMA INTEGRAL DE GESTIÓN}

Se propone una matriz formada por los cinco grandes grupos de procesos (Fig.4)

\begin{tabular}{|c|c|c|c|c|}
\hline 1. INICIO & 2. PLANIFICACIÓN & 3. EJECUCIÓN & $\begin{array}{l}\text { 4. SEGUIMIENTOY } \\
\text { CONTROL }\end{array}$ & 5. CIERRE \\
\hline \multirow[t]{2}{*}{$\begin{array}{l}\text { - Desarrollar el Acta de } \\
\text { Constitución y su } \\
\text { alineación con el plan } \\
\text { estratégico y } \\
\text { objetivos. } \\
\text { - Lista de Interesados } \\
\text { de Proyectos. } \\
\text { - Revisión de } \\
\text { Expediente Técnico. }\end{array}$} & $\begin{array}{l}\text { - Enunciado del Alcance. } \\
\text { - EDT de los proyectos. } \\
\text { - Organigrama } \\
\text { - Cronograma. } \\
\text { - Presupuesto } \\
\text { - Plan de gestión de } \\
\text { Adquisiciones } \\
\text { - Matriz de } \\
\text { Comunicaciones } \\
\text { - Matriz de Riesgos } \\
\text { - TDR Contratistas } \\
\text { - TDR supervisión }\end{array}$ & $\begin{array}{l}\text { - Actas de Reunión. } \\
\text { - Buena pro: se tiene } \\
\text { a los proveedores de } \\
\text { construcción y } \\
\text { supervisión } \\
\text { - Contratos del } \\
\text { contratista } \\
\text { - Contratos del } \\
\text { supervisor } \\
\text { - Efectuar } \\
\text { Adquisiciones }\end{array}$ & $\begin{array}{l}\text { - Solicitudes de } \\
\text { - Rembio. } \\
\text { - Aprobación de Polémicas de } \\
\text { Solicitudes de cambio } \\
\text { - Lista de Problemas } \\
\text { - Revisión de informes } \\
\text { mensuales. } \\
\text { - Controlar } \\
\text { - Adquisiciones } \\
\text { - Lecciones Aprendidas }\end{array}$ & $\begin{array}{l}\text { - Cierre Técnico } \\
\text { - Cierre } \\
\text { Administrativo y } \\
\text { Financiero. } \\
\text { - Listado de } \\
\text { Lecciones } \\
\text { Aprendidas } \\
\text { - Cerrar } \\
\text { Adquisiciones }\end{array}$ \\
\hline & & $\begin{array}{l}\text { - Plan de Trabajo } \\
\text { - Desarrollo del Plan } \\
\text { de Ejecución del } \\
\text { Proyecto } \\
\text { - Valorizaciones. } \\
\text { - Cronograma } \\
\text { detallado lookahead } \\
\text { (planificar con } \\
\text { anticipación) } \\
\text { - Registro de PPC } \\
\text { (Porcentaje de } \\
\text { planificación } \\
\text { cumplida) } \\
\text { - Avance de obra }\end{array}$ & $\begin{array}{l}\text { - Solicitudes de Cambio. } \\
\text { - Informe de desempeño. } \\
\text { - Informe Semanal } \\
\text { SPI (índice de } \\
\text { desempeño del } \\
\text { cronograma), CPI (Índice } \\
\text { de desempeño de } \\
\text { costos) } \\
\text { - Informe Mensual } \\
\text { - Inspección de calidad } \\
\text { - Recursos humanos } \\
\text { - Lecciones Aprendidas. } \\
\text { - Inspecciones de calidad } \\
\text { - Informes mensuales. } \\
\text { SPI, CPI } \\
\text { - Lecciones aprendidas }\end{array}$ & $\begin{array}{l}\text { - Cierre Técnico } \\
\text { - Cierre } \\
\text { Administrativo y } \\
\text { Financiero. } \\
\text { - Registro de } \\
\text { Lecciones } \\
\text { Aprendidas }\end{array}$ \\
\hline & & $\begin{array}{l}\text { - Desarrollo del plan } \\
\text { de Trabajo de } \\
\text { supervisión } \\
\text { - EDT } \\
\text { - Organigrama } \\
\text { - Validación de } \\
\text { avance Y } \\
\text { Valorización } \\
\end{array}$ & $\begin{array}{l}\text { - Registro de Solicitudes } \\
\text { de cambio } \\
\text { - Inspecciones de calidad } \\
\text { - Informes mensuales. } \\
\text { SPI, CPI } \\
\text { - Lecciones aprendidas } \\
\text { - Registro de Solicitudes } \\
\text { de cambio }\end{array}$ & $\begin{array}{l}\text { - Cierre Técnico } \\
\text { - Cierre } \\
\text { Administrativo y } \\
\text { Financiero. } \\
\text { - Registro de } \\
\text { Lecciones } \\
\text { Aprendidas }\end{array}$ \\
\hline
\end{tabular}

1. CLIENTE: GERENCIA DE INFRAES TRUCTURA

2. EMPRESA: CONTRATISTA

3. EMPRESA: SUPERVISIÓN

Fig. 1: Matriz de los Procesos en el Ciclo de vida del Proyecto 
Y constó de los siguientes instrumentos elaborados como propuesta:

1. Matriz de entregables del SIG en el ciclo de vida del proyecto

2. Procesos del sistema integral de gestión para mejorar la gestión de proyectos de saneamiento

3. Grupo de procesos de planificación del sistema integral de gestión

4. Grupo de procesos de ejecución del sistema integral de gestión

5. Grupo de procesos de monitoreo y control del sistema integral de gestión

6. Grupo de procesos de cierre del sistema integral de calidad

\section{DISCUSIÓN}

Se desarrolló un Sistema integral de Gestión que permite gerenciar un proyecto de saneamiento desde el punto de vista del cliente oficina de infraestructura, a través de formatología debidamente estructurada, durante el ciclo de vida del proyecto desde que se tiene el expediente técnico, se compatibiliza hasta su ejecución y cierre. En el trabajo se diseñaron formatos de aplicación según el ciclo de vida de un proyecto de saneamiento que son el inicio, planificación, ejecución, seguimiento y control y cierre, para llevar el efectivo control de los proveedores de supervisión y construcción. Se simuló el Sistema integral de Gestión en un proyecto de saneamiento en la provincia de Tacna departamento de Tacna localidad de Tacna por parte de la EPS TACNA, y se obtuvo una documentación estructurada que permitirá llevar un mejor control de sus proveedores para proyectos futuros, aumentado la capacidad de gestión de la entidad contratante. Se formularon postulados para los TDRs de la supervisión y contratista en obras de saneamiento para una adecuada administración del contrato. Se obtuvieron respuestas concluyentes a través de las encuestas a 30 profesionales involucrados en la elaboración de TDRs de supervisión y construcción (76\%), también desempeñaron como supervisores $(77 \%)$ y de construcción (90\%) , todos ellos en proyectos de saneamiento. Es importante efectuar la evaluación post obra, para poder monitorear si el proyecto cumplió con sus objetivos. En el aplicativo efectuado en el presente estudio se pudo observar que se logró obtener un $50 \%$ de reducción de los problemas en la ejecución de la obra. Asimismo, el Sistema integral de Gestión es una guía flexible a la cual se pueden y deben adaptar, incluir más formatos y/o herramientas propias de cada entidad e incluso excluir formatos que se crean no convenientes para el tamaño en alcance, costo, tiempo del proyecto de saneamiento. Los formatos de gestión son adaptados a la necesidad de los proyectos de la región Tacna y en una entidad pública que por lo general no posee modelos ni sistemas de gestión, en caso se desee aplicar el Sistema integral de Gestión para proyectos regionales de gran envergadura. El Sistema puede satisfacer todas esas necesidades requeridas por lo que se recomienda para estos casos como una guía de apoyo. En base a este trabajo se podrían abrir futuras líneas de investigación acerca de la gestión pública desde el punto de vista de gerenciador que aplique herramientas de control estándar que permita un efectivo control y planeamiento de sus proyectos.

\section{BIBLIOGRAFÍA}

Alcina, Jorge. (2006). "Como Cerrar Correctamente un Proyecto", Artículo, PMI, Venezuela.

Cameron, K.S., and Whetten, D.A. (1983). "Organisational Effectiveness: A Comparison of Multiple Models", Articulo, USA.

Farfan Cruz, J, F. J. (2005). Modelo Básico de Gestión de proyectos de abastecimeinto de agua potable y saneamiento ante el plan internacional aplicado a la comunidad de Trinidad.

Hernández Rodríguez, E. R. (2013). Análisis de la Sostenibilidad de los Operadores de Sistemas de Agua Potable y Saneamiento en el municipio de Suchitoto, departamento de Cuscatlán. (engd). Universidad de El Salvador. Recuperado a partir de http://ri.ues.edu.sv/4655/

LEY N ${ }^{\circ} 30225$ y D.S. N ${ }^{\circ} 350-2015-E F$. (s. f.). Ley de Contrataciones del Estado y su Reglamento.

PMBOK del P.M.I. (2013). A Guide to the Project Management Body of Knowledge (PMBOK® Guide) — Fifth edition @2013 Project Management Institute.

Recibidos: 17 / $01 / 17$

Aceptado para publicación: 09 / 06 / 17 